\title{
Effect of Chinese herbal medicine extracts on cell-mediated immunity in a rat model of tuberculosis induced by multiple drug-resistant bacilli
}

\author{
JUN LU ${ }^{1}$, SONG YE ${ }^{1}$, RUI QIN ${ }^{1}$, YUN DENG $^{1}$ and CHAO-PIN LI ${ }^{2}$ \\ ${ }^{1}$ Department of Pathogen Biology and Immunology, Anhui University of Science and Technology, \\ Huainan 232001; ${ }^{2}$ Department of Pathogen Biology, Wannan Medical College, Wuhu 241002, P.R. China
}

Received January 23, 2013; Accepted May 15, 2013

DOI: $10.3892 / \mathrm{mmr} .2013 .1491$

\begin{abstract}
Drug-resistant Mycobacterium tuberculosis poses a major threat to public health. The present study aimed to investigate the effects of Radix Ranunculi Ternati, Radix Sophorae Flavescentis, Prunella Vulgaris L. and Stellera Chamaejasme L. extracts on cell-mediated immunity in a rat model of tuberculosis (TB) induced by multiple drugresistant bacilli. The bacterium was isolated from patients infected with pulmonary tuberculosis. The immunological response in humans following infection with Mycobacterium tuberculosis involves a number of cytokines, including IFN- $\gamma$ and IFN- $\alpha$, which are important for killing intracellular micro-organisms. T helper type 2 (Th2) cells express numerous cytokines, including IL-4 and IL-10, which mainly participate in humoral immunity and induce the phagocytosis of extracellular bacteria and parasites. In the present study, rats were infected with multiple drug-resistant TB (MDR-TB) in order to establish an MDR-TB model. Peripheral blood mononuclear cells (PBMCs) were isolated and cultivated, and the serum levels of IFN- $\gamma$, IL-4, IL-10 and IL-12 were examined by ELISA. The mRNA expression levels of certain cytokines in PBMCs were additionally detected using RT-PCR. The serum levels of IFN- $\gamma$ in the Radix Ranunculi Ternati, Radix Sophorae Flavescentis, Prunella Vulgaris L. and Stellera Chamaejasme L. groups were $2.01 \pm 0.73,1.92 \pm 0.56,1.98 \pm 0.67$ and $1.94 \pm 0.59 \mathrm{pg} / \mathrm{ml}$; IL-4 levels were $6.01 \pm 1.46,6.12 \pm 1.35$, $6.47 \pm 1.46$ and $6.15 \pm 1.44 \mathrm{pg} / \mathrm{ml}$; IL-10 levels were $12.09 \pm 3.07$, $12.45 \pm 4.01,12.13 \pm 3.43$ and $12.54 \pm 3.78 \mathrm{pg} / \mathrm{ml}$; and IL-12 levels were $2.99 \pm 0.89,2.75 \pm 0.84,3.02 \pm 0.86$ and $2.89 \pm 0.75 \mathrm{pg} / \mathrm{ml}$,
\end{abstract}

Correspondence to: Dr Jun Lu, Department of Pathogen Biology and Immunology, School of Medicine, Anhui University of Science and Technology, 25 Dongshanzhong Road, Huainan, Anhui 232001, P.R. China

E-mail: cfdxlujun@126.com

Key words: Radix Ranunculi Ternati, Radix Sophorae Flavescentis, Prunella Vulgaris L., Stellera Chamaejasme L., extract of Chinese herbal medicine, multiple drug-resistant Bacillus tuberculosis, cytokine, immunological function respectively. These differences were significant compared with the model group $(\mathrm{P}<0.05)$. RT-PCR analysis revealed a significant increase in the levels of IFN- $\gamma$ and IL-12, and a significant decrease in the mRNA levels of IL-4 and IL-10 $(\mathrm{P}<0.05)$. These results indicated that the extracts of Radix Ranunculi Ternati, Radix Sophorae Flavescentis, Prunella Vulgaris L. and Stellera Chamaejasme L. are capable of enhancing cell-mediated immunity in rats by upregulating the levels of genetic transcription. This may explain the observed therapeutic effect of Chinese herbal medicines in the treatment of MDR-TB.

\section{Introduction}

Tuberculosis (TB) is a chronic infectious disease caused by the bacterium Mycobacterium tuberculosis, and it remains a significant public health risk worldwide. The pestilence of TB in the Cosmopolitan population has been eased with the introduction of anti-TB and -HIV drugs during the 1980s $(1,2)$. However, the management of TB incidence has become more difficult with the emergence of multiple drug-resistant (MDR) and mycobacterial cell wall-deficient strains. Therefore, there is an urgent need to develop new anti-TB agents that are effective in the treatment of active and latent TB, and MDR-TB. Thus, the identification of potential therapeutic agents to prevent and treat MDR-TB infection by natural drug screening is important. Radix Ranunculi Ternati, Radix Sophorae Flavescentis, Prunella Vulgaris L. and Stellera Chamaejasme L. are common traditional Chinese herbal medicines with heat-clearing and detoxifying effects (3-5). In recent years, several studies have demonstrated that these extracts possess anti-Mycobacterium tuberculosis activity in vitro (6-8). In the present study, we examined the therapeutic effects of traditional Chinese herbal medicines for the treatment of MDR-TB. The effect of these extracts on cell-mediated immunity in rats was also examined, to provide preliminary data for the clinical application of Chinese herbal medicines in the treatment of MDR-TB.

\section{Materials and methods}

Animals. A total of 60 adult, male Kunming rats weighing 18-22 $\mathrm{g}$ were purchased from the Center of Experimental 
Animals (Nanjing University, Nanjing, China) and bred in the university facilities. All animal experiments were performed in accordance with the Chinese laws for animal protection and in adherence with the experimental guidelines and procedures approved by the Institutional Animal Care and Use Committee (IACUC) of Nanjing Medical University for the use of laboratory animals (permit number: NJMU 09-1107) and The Ethical Review Committee of Nanjing Medical University for the use of laboratory animals.

MDR-TB models. The rats were randomly divided into six groups, each containing ten rats: The normal group (fed daily on a standard diet); the model group (drenched with $5 \mathrm{ml}$ sodium chloride and then fed on a standard diet); and four groups that were each treated with one of the extracts of Chinese herbal medicines (drenched with $5 \mathrm{ml}$ of extract daily at a concentration of $200 \mathrm{mg} / \mathrm{kg}$, and fed on a standard diet for 4 weeks). The rats were then sacrificed and serum was collected following sterilization (9). The purified MDR-TB colony was prepared in a $1 \mathrm{mg} / \mathrm{ml}$ bacterial suspension with sterile saline. Each rat was injected with $2 \mathrm{ml}$ of suspension via the tail vein (10).

Preparation of the Chinese herbal medicine extracts. The extracts of Radix Ranunculi Ternati, Radix Sophorae Flavescentis, Prunella Vulgaris L. and Stellera Chamaejasme L. were prepared with distilled water. The solvent was reflowed, extracted and filtrated with $20 \%$ ethanol to remove any impurities. The extracts were then concentrated and freeze-dried to obtain the ethanol extract (11).

ELISA analysis. The serum levels of IFN- $\gamma$, IL-4, IL-10 and IL-12 were quantified using the double antibody sandwich ELISA kit (Jingmei Biotech, Co., Ltd., Shenzhen, Guangdong, China). The assay was performed according to the manufacturer's instructions. Briefly, $150 \mu 1$ sample was added to microtiter plates, which were coated with the respective monoclonal antibody. The reaction plate was then mixed at $37^{\circ} \mathrm{C}$ for $120 \mathrm{~min}$ to fully integrate the antigen and antibody. Following washing, streptavidin antibody fluid labeled with $100 \mu 1$ horseradish peroxidase was kept at $37^{\circ} \mathrm{C}$ for $60 \mathrm{~min}$ and added to $100 \mu \mathrm{lo}$-phenylenediamine dihydrochloride at $37^{\circ} \mathrm{C}$ for 5-10 min in the dark. The optical density was measured at a wavelength of $492 \mathrm{~nm}$ using an ELISA microplate reader (Bio-Tek Instruments, Inc., Winooski, VT, USA). The serum concentrations were calculated and expressed as $\mathrm{pg} / \mathrm{ml}$.

Isolation and culture of PBMCs. The animals were sacrificed following the removal of blood from the femoral artery. PBMCs were isolated by density gradient centrifugation. The cells were routinely grown in RPMI-1640 medium supplemented with $10 \% \mathrm{FBS}, 50 \mathrm{U} / \mathrm{ml}$ penicillin and $50 \mathrm{mg} / \mathrm{ml}$ streptomycin. Following resuspension, $0.5 \mathrm{ml}$ cell suspension was plated out in 24-well culture plates at a density of $1 \times 10^{6}$ cells $/ \mathrm{ml}$ and cells were cultured in an incubator at $37^{\circ} \mathrm{C}$ and $5 \% \mathrm{CO}_{2}$. The medium was cultivated after $24 \mathrm{~h}$.

Primer construction. The primer sequences used in the study were as follows: IFN- $\gamma$ forward, 5'-ACAATGAACGCT ACACACTGC-3' and reverse, 5'-CGAATCAGCAGCGA
CTCCTT-3', product size 456 bp; IL-12 forward, 5'-GCTAACCATCTCCTGGTTTGC-3' and reverse, 5'-CTTTCCAGAGCCTATGACTCC-3', product size 390 bp; IL-4 forward, 5'-CTCACAGCAACGAAGAACAC-3' and reverse, 5'-GGCTCAGTACTACGAGTAATCC-3', product size $267 \mathrm{bp}$; IL-10 forward, 5'-CTTTCAAATGAA GGATCAGC-3' and reverse, 5'-ATGTCAAAC TCACTCATGGC-3', product size $327 \mathrm{bp}$; and GLS forward, 5'-GGGAAGCTCCATAAATGTCACCT-3' and reverse, 5'-GGTTTAGATCGGCACAT-3', product size 405 bp. For the housekeeping gene GAPDH, the primers used were forward, 5'-GAAGGTGAAGGTCGGAGT-3' and reverse, 5'-GAAGATGGTGATGGGATTTA-3', product size $320 \mathrm{bp}$. The above primers were synthesized by Sangon Biotech, Co., Ltd. (Shanghai, China).

RT-PCR analysis. Total RNA was isolated from PBMCs using TRIzol (Invitrogen Life Technologies, Carlsbad, CA, USA) according to the manufacturer's instructions, and collected using a one-step method at a concentration of $200 \mu \mathrm{g} / \mathrm{ml}$. The total volume used for reverse transcription was $50 \mu 1$ and the procedure was performed using the SYBR-Green PCR kit (Applied Biosystems, Foster City, CA, USA) according to the manufacturer's instructions. The RT-PCR reaction conditions were as follows: RT reaction at $50^{\circ} \mathrm{C}$ for $30 \mathrm{~min}$; RTase inactivation at $94^{\circ} \mathrm{C}$ for $5 \mathrm{~min}, 9^{\circ} \mathrm{C}$ for $30 \mathrm{sec}, 55^{\circ} \mathrm{C}$ for $90 \mathrm{sec}$ and $72^{\circ} \mathrm{C}$ for $30 \mathrm{sec}$ for 35 cycles in total, followed by maintenance at $72^{\circ} \mathrm{C}$ for $10 \mathrm{~min}$. The amplified products $(5 \mu \mathrm{l})$ were separated by electrophoresis on $1.5 \%$ agarose gel, and were then imaged and analyzed using ImageMaster TotalLab software (GE Healthcare Biosciences, Pittsburgh, PA, USA). The ratios of the target genes and the internal reference gene (GAPDH) were used to determine the relative expression levels of the target genes.

Statistical analysis. Data are expressed as the mean $\pm \mathrm{SD}$, and were analyzed using SPSS 12.0 software (SPSS, Inc., Chicago, IL, USA). One-way ANOVA was used for statistical analysis to determine differences between groups. $\mathrm{P}<0.05$ was considered to indicate a statistically significant result.

\section{Results}

ELISA analysis. The serum levels of IFN- $\gamma$, IL-4, IL-10 and IL-12 were examined using ELISA (Table I). The levels of IFN- $\gamma$ and IL-12 were significantly decreased in the model group compared with the normal group, and the levels of IL-4 and IL-10 were significantly increased $(\mathrm{P}<0.01)$. The levels of IFN- $\gamma$, IL-12, IL-4 and IL-10 in the Radix Ranunculi Ternati group demonstrated a significant increase compared with the model group $(\mathrm{P}<0.05)$. In the Radix Sophorae Flavescentis group, the levels of IFN- $\gamma$ and IL- 12 were significantly increased $(\mathrm{P}<0.05)$, and the levels of IL-4 and IL-10 were significantly decreased compared with the model group $(\mathrm{P}<0.05)$. In the Prunella Vulgaris L. group, the levels of IFN- $\gamma$, IL-10 and IL-12 were significantly increased compared with the model group $(\mathrm{P}<0.05)$; however, no significant changes in IL-4 levels were detected. In the Stellera Chamaejasme L. group, the levels of IL-4, IL-10 and IL-12 were significantly increased $(\mathrm{P}<0.05)$ and the levels of IFN- $\gamma$ were significantly decreased $(\mathrm{P}<0.05)$ compared with the model group. 
Table I. Serum levels of IFN- $\gamma$, IL-4, IL-10 and IL-12 in different groups of rats, as determined by ELISA.

\begin{tabular}{|c|c|c|c|c|c|}
\hline Group & Number & $\mathrm{IFN}-\gamma(\mathrm{pg} / \mathrm{ml})$ & $\mathrm{IL}-12(\mathrm{pg} / \mathrm{ml})$ & IL-4 (pg/ml) & $\mathrm{IL}-10(\mathrm{pg} / \mathrm{ml})$ \\
\hline Normal & 10 & $2.24 \pm 0.62$ & $3.79 \pm 0.92$ & $5.58 \pm 1.43$ & $11.23 \pm 2.08$ \\
\hline Model & 10 & $1.18 \pm 0.38^{\mathrm{a}}$ & $2.19 \pm 0.57^{\mathrm{a}}$ & $8.15 \pm 2.24^{\mathrm{a}}$ & $16.10 \pm 2.21^{\mathrm{a}}$ \\
\hline Radix Ranunculi Ternati & 10 & $2.01 \pm 0.73$ & $2.99 \pm 0.89^{b}$ & $6.01 \pm 1.46$ & $12.09 \pm 3.07^{\mathrm{b}}$ \\
\hline Radix Sophorae Flavescentis & 10 & $1.92 \pm 0.56^{\mathrm{b}}$ & $2.75 \pm 0.84^{\mathrm{b}}$ & $6.12 \pm 1.35^{\mathrm{b}}$ & $12.45 \pm 4.01^{\mathrm{b}}$ \\
\hline Prunella Vulgaris L. & 10 & $1.98 \pm 0.67^{\mathrm{b}}$ & $3.02 \pm 0.86^{\mathrm{b}}$ & $6.47 \pm 1.46^{\mathrm{b}}$ & $12.13 \pm 3.43^{\mathrm{b}}$ \\
\hline Stellera Chamaejasme L. & 10 & $1.94 \pm 0.59^{b}$ & $2.89 \pm 0.75^{b}$ & $6.15 \pm 1.44^{\mathrm{b}}$ & $12.54 \pm 3.78^{b}$ \\
\hline
\end{tabular}

Data are presented as the mean $\pm \mathrm{SD} .{ }^{\mathrm{a}} \mathrm{P}<0.01$ vs. the normal group; ${ }^{\mathrm{b}} \mathrm{P}<0.05$ vs. the model group.

Table II. mRNA levels of IFN- $\gamma$, IL-4, IL-10, IL-12 and GLS in rat PBMCs, as determined by RT-PCR.

\begin{tabular}{|c|c|c|c|c|c|}
\hline Group & $\begin{array}{l}\text { IFN- } \gamma / \text { GAPDH } \\
\text { (gray ratio) }\end{array}$ & $\begin{array}{l}\text { IL-12/GAPDH } \\
\text { (gray ratio) }\end{array}$ & $\begin{array}{l}\text { IL-4/GAPDH } \\
\text { (gray ratio) }\end{array}$ & $\begin{array}{l}\text { IL-10/GAPDH } \\
\text { (gray ratio) }\end{array}$ & $\begin{array}{l}\text { GLS/GAPDH } \\
\text { (gray ratio) }\end{array}$ \\
\hline Normal & $0.31 \pm 0.06$ & $0.78 \pm 0.10$ & $0.45 \pm 0.05$ & $0.63 \pm 0.08$ & $0.22 \pm 0.04$ \\
\hline Model & $0.18 \pm 0.05^{\mathrm{a}}$ & $0.59 \pm 0.08^{\mathrm{a}}$ & $0.61 \pm 0.06^{\mathrm{a}}$ & $0.99 \pm 0.12^{\mathrm{a}}$ & $0.14 \pm 0.04^{\mathrm{a}}$ \\
\hline Radix Ranunculi Ternati & $0.27 \pm 0.06^{\mathrm{b}}$ & $0.75 \pm 0.09^{b}$ & $0.50 \pm 0.05^{\mathrm{b}}$ & $0.69 \pm 0.08^{b}$ & $0.21 \pm 0.05^{\mathrm{c}}$ \\
\hline Radix Sophorae Flavescentis & $0.25 \pm 0.07^{\mathrm{b}}$ & $0.64 \pm 0.09$ & $0.52 \pm 0.06^{\mathrm{b}}$ & $0.83 \pm 0.11$ & $0.17 \pm 0.05$ \\
\hline Prunella Vulgaris L. & $0.26 \pm 0.06^{\mathrm{b}}$ & $0.71 \pm 0.11^{\mathrm{b}}$ & $0.58 \pm 0.05$ & $0.70 \pm 0.10^{\mathrm{b}}$ & $0.18 \pm 0.04$ \\
\hline Stellera Chamaejasme L. & $0.22 \pm 0.07$ & $0.68 \pm 0.10^{\mathrm{b}}$ & $0.55 \pm 0.06^{\mathrm{b}}$ & $0.72 \pm 0.12$ & $0.19 \pm 0.04^{\mathrm{b}}$ \\
\hline
\end{tabular}

Data are presented as the mean $\pm \mathrm{SD}$. ${ }^{\mathrm{a}} \mathrm{P}<0.01$ vs. the normal group; ${ }^{\mathrm{b}} \mathrm{P}<0.05$ vs. the model group; ${ }^{\mathrm{c}} \mathrm{P}<0.01$ vs. the model group. GLS, granulysin; PBMCs, peripheral blood mononuclear cells.

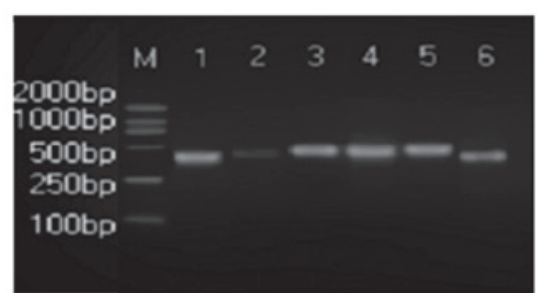

IFN- $\gamma$

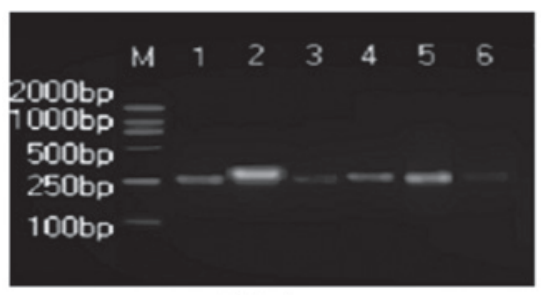

IL-4

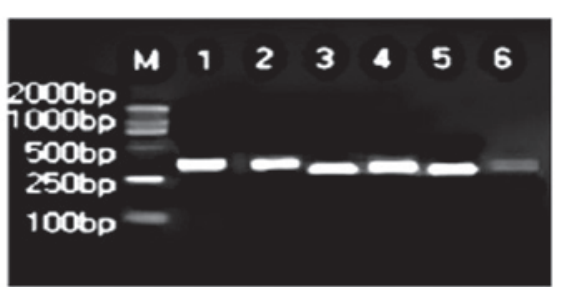

IL-12

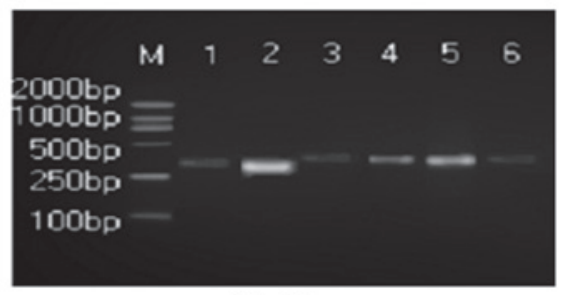

IL-10

Figure 1. RT-PCR analysis for the effects of the Radix Ranunculi Ternati extract on mRNA levels. Lane M, DL2000 marker (2000, $1000,500,250$ and 100 bp); lane 1, normal control group; lane 2, model group; and lanes 3-6, Radix Ranunculi Ternati group.

RT-PCR analysis. RT-PCR was used to examine the mRNA expression levels of IFN- $\gamma$, IL-4, IL-10 and IL-12 in the PBMCs of rats (Table II). Compared with the normal group, the mRNA levels of IFN- $\gamma$ and IL-12 were significantly decreased in the model group, and IL-4 and IL-10 levels were significantly increased $(\mathrm{P}<0.01)$. The mRNA expression levels of IFN- $\gamma$, IL-12, IL-4 and IL-10 in the PBMCs of the
Radix Ranunculi Ternati group were significantly increased compared with the model group $(\mathrm{P}<0.05$; Fig. 1$)$. The levels of IFN- $\gamma$ and IL-4 in PBMCs of the Radix Sophorae Flavescentis group were significantly increased $(\mathrm{P}<0.05)$, while IL-10 and IL-12 levels were significantly decreased compared with the model group $(\mathrm{P}<0.05$; Fig. 2). The mRNA expression levels of IFN- $\gamma$ and IL-12 in the Prunella Vulgaris L. group increased 


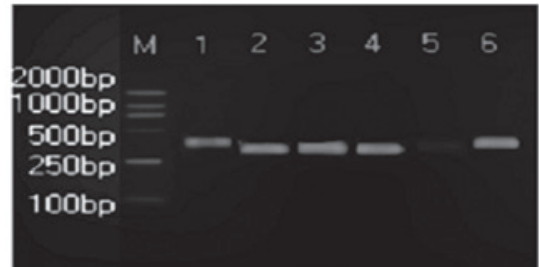

IFN- $\gamma$

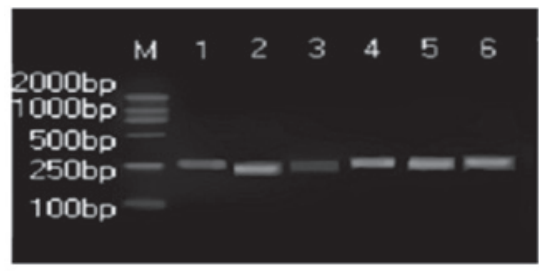

IL-4

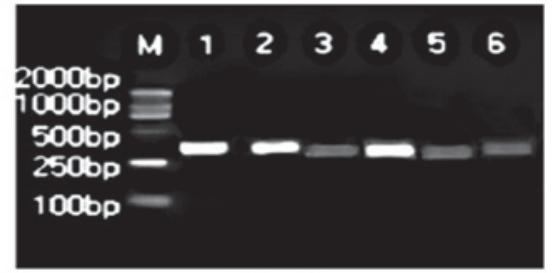

IL-12

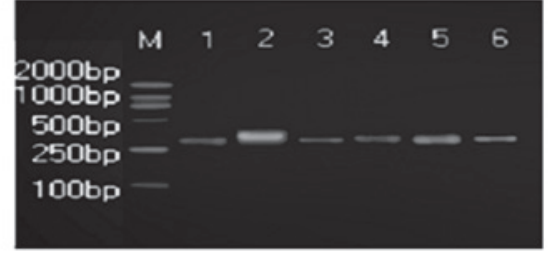

IL-10

Figure 2. RT-PCR analysis for the effects of the Radix Sophorae Flavescentis extract on mRNA levels. Lane M, DL2000 marker (2000, 1000, 500, 250 and 100 bp); lane 1, normal control group; lane 2, model group; and lanes 3-6, Radix Sophorae Flavescentis group.

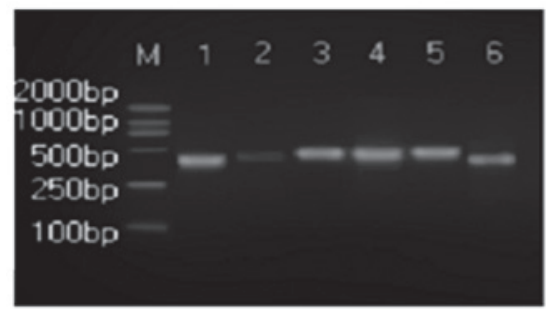

IFN- $\gamma$

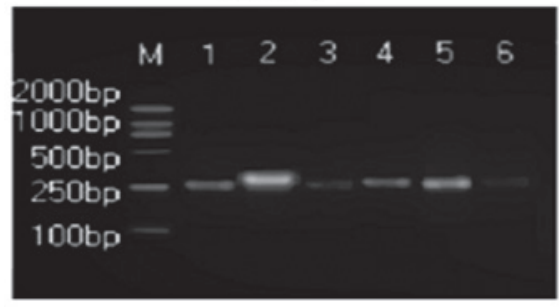

IL-4

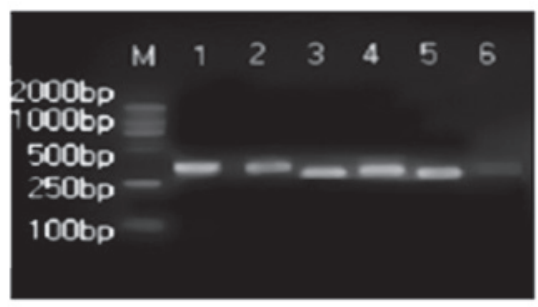

IL-12

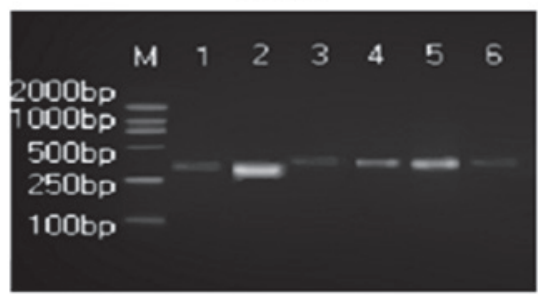

IL-10

Figure 3. RT-PCR analysis for the effects of the Prunella Vulgaris L. extract on mRNA levels. Lane M, DL2000 marker (2000, 1000, 500 , 250 and 100 bp); lane 1, normal control group; lane 2, model group; and lanes 3-6, Prunella vulgaris L.group..

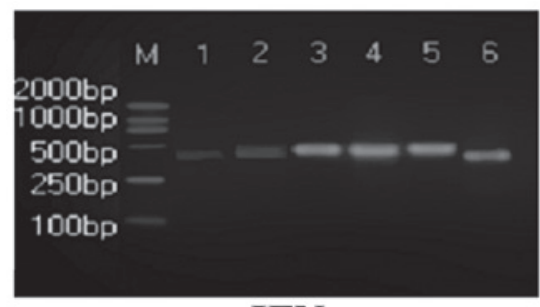

IFN- $\gamma$

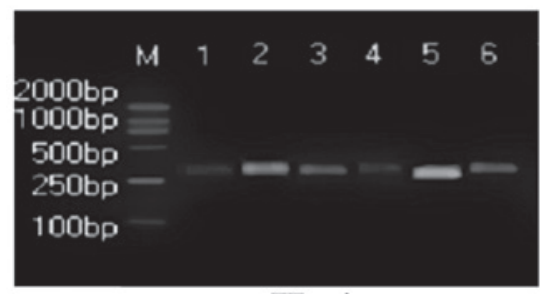

IL-4

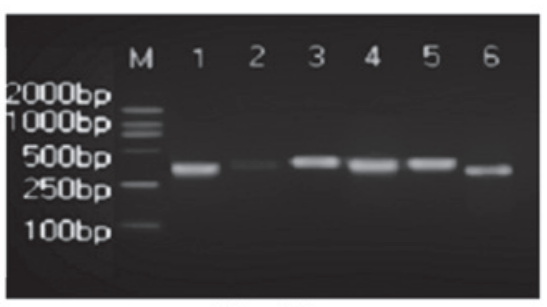

IL-12

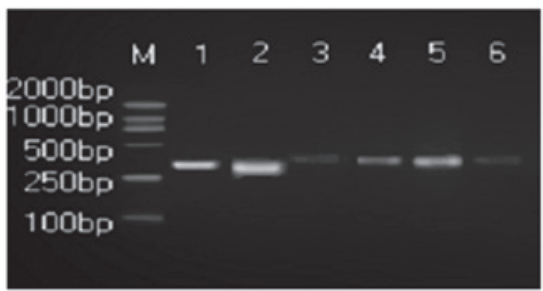

IL-10

Figure 4. RT-PCR analysis for the effects of the Stellera Chamaejasme L. extract on mRNA levels. Lane M, DL2000 marker (2000, 1000, 500, 250 and 100 bp); lane 1, normal control group; lane 2, model group; and lanes 3-6, Stellera Chamaejasme L. group. 
markedly, and the levels of IL-4 and IL-10 decreased significantly compared with the model group $(\mathrm{P}<0.05)$; however, no evident change in IL-4 levels were observed $(\mathrm{P}<0.05$; Fig. 3). IL-4, IL-10 and IL-12 levels in PBMCs of the Stellera Chamaejasme L. group increased significantly $(\mathrm{P}<0.05)$ and IFN- $\gamma$ levels decreased markedly compared with the model group ( $\mathrm{P}<0.05$; Fig. 4). Granulysin (GLS), a type of polypeptide whose expression is induced by cytotoxic $\mathrm{T}$ lymphocytes (CTLs) and natural killer cells during late activation, is capable of killing Mycobacterium tuberculosis. The mRNA expression level of GLS is one of the markers that demonstrates the molecular effectiveness of cells that kill intracellular bacteria. Compared with the model group, the mRNA levels of GLS increased significantly $(\mathrm{P}<0.05$ or $\mathrm{P}<0.01)$.

\section{Discussion}

Radix Ranunculi Ternati is a prescription medicine that has been reported in the Chinese pharmacopoeia to facilitate detumescence. Radix Ranunculi Ternati may also possess anticancer properties; however, the mechanism by which these effects are exerted has yet to be fully elucidated (12). Radix Sophorae Flavescentis has diuretic, anti-inflammatory, antiviral, anticancer and antifibrotic properties, and may also be used to treat a number of immunological diseases $(13,14)$. Prunella Vulgaris L. has been shown to improve eyesight and eliminate stagnation by partially inhibiting tumor cell proliferation in vivo, and it induces its antitumor effects by regulating several cell signal transduction pathways, which stimulates the activation of macrophages (15-17). Stellera Chamaejasme L. possesses antitumor, antiviral and immunological properties (18).

Mycobacterium tuberculosis undergoes an initial replicative phase inside alveolar macrophages. Following this stage, it then enters a non-replicative, drug-resistant state of dormancy. It is able to survive in this dormant state for decades until the immune system of the host is weakened, at which point the bacterium reactivates and causes the infectious disease. The immunological response to Mycobacterium tuberculosis infection in humans is predominantly mediated by Th1 cells. Th1 cells predominantly secrete IFN- $\gamma$ and INF- $\alpha$, which stimulate macrophages to kill intracellular micro-organisms. Th2 cells mainly participate in humoral immunity, and secrete IL-4, IL-10 and several other cytokines that stimulate the phagocytosis of extracellular bacteria and parasites. Mycobacterium tuberculosis is a facultative intracellular bacterium that parasitizes macrophages. The immune response to Mycobacterium tuberculosis infection is mainly exerted by $\mathrm{T}$ cells and antigen presentation by $\mathrm{MHC}$ class II molecules. It is well known that IFN- $\gamma$ produced by TB-specific $\mathrm{CD}^{+} \mathrm{T}$ cells (Th1) is able to activate macrophages, secreting IL-12 to promote the differentiation of Th0 cells to Th1 cells, further expanding the Th1 cell immune response. Thus, Th1 cells possess an important anti-TB protective role; however, the cytokines secreted by Th 2 cells, including IL-4 and IL-10, are also secreted by Mycobacterium tuberculosis (19-21). Therefore, the regulation of immune homeostasis by Th1/Th2 cells may affect the type of immunological response initiated by the body's immune system.

In the present study, we demonstrated that infection with Mycobacterium tuberculosis significantly affects the serum levels of cytokines in rats. The levels of IFN- $\gamma$ and IL-12 in the model group were markedly decreased and the levels of IL-4 and IL-10 were significantly increased compared woth the normal group (Table I). Experimental results demonstrated that the occurrence of TB is correlated with the balance of Th1/Th2 cell responses. The four extracts of Chinese herbal medicines used in this study, particularly Radix Ranunculi Ternati, significantly enhanced the cell-mediated immunological response of rats infected by MDR-TB through the adjustment the Th1/Th2 balance. Therefore, these extracts may be important in the treatment of TB.

Furthermore, results of the RT-PCR analysis demonstrated that the mRNA expression levels of IFN- $\gamma$, IL-12 and GLS in the PBMCs of MDR-TB-infected rats were significantly increased, and the levels of IL-4 and IL-10 were significantly decreased compared with the normal group (Table II); these differences were significant $(\mathrm{P}<0.05$ or $\mathrm{P}<0.01)$. The four extracts of Chinese herbal medicines were found to be capable of stimulating the expression of IFN- $\gamma$, IL-12 and GLS mRNA and downregulating the expression of IL-4 and IL-10. The results demonstrated the regulation of cellular immunity of the extracts is accomplished at the level of gene transcription. It was also demonstrated that Radix Ranunculi Ternati is able to stimulate the expression of GLS mRNA, thus enhancing the sterilization ability of CTLs, so as to kill Mycobacterium tuberculosis (22).

In conclusion, appropriate concentrations of the Radix Ranunculi Ternati, Radix Sophorae Flavescentis, Prunella Vulgaris L. and Stellera Chamaejasme L. extracts are capable of enhancing cell-mediated immunity in rats infected by MDR-TB, providing an experimental basis for the clinical treatment of TB using these extracts. However, further research is required with regard to the role of other cytokines.

\section{Acknowledgements}

This study was supported by grants from the National Natural Science Foundation of China (NSFC 81172778) and the Natural Science Foundation of Anhui Province (KJ2010A087).

\section{References}

1. Telenti A and Iseman M: Drug-resistant tuberculosis: what do we do now? Drugs 59: 171-179, 2000.

2. Du Toit LC, Pillay V and Danckwerts MP: Tuberculosis chemotherapy: current drug delivery approaches. Respir Res 7: 118, 2006.

3. Zhang ZL, Wu XJ, Wang L and Zhang HX: Study on immunocompetence of active constituent of Radix Ranunculi Ternati. Zhong Hua Zhong Yi Yao Xue Hui She 22: 120-122, 2007 (In Chinese).

4. Fu XR, Li JC and Zhang MZ: Advances in modern research on prunella spike. Zhong Yi Yan Jiu Bian Ji Bu 18: 60-62, 2005 (In Chinese).

5. Ji RL, Xia SH and Li F: Oxymatrine inhibits MMP-2 expression and reduces cell invasion in human pancreatic carcinoma cell line SW1990. Shi Jie Hua Ren Xiao Hua Za Zhi Bian Ji Bu 19: 19-24, 2011 (In Chinese).

6. Wu HD and Wang L: Research of antituberculotic. Medical Review 13: 475-478, 2007.

7. Lu J, Ye S, Deng Y, et al: Effects of four extracts of Chinese herbal medicines on cellular immunity in rats induced by multiple drugs resistant bacillus tuberculosis among pneumoconiosis patients complicated with tuberculosis. Zhong Hua Wei Sheng Wu Xue He Mian Yi Xue Za Zhi Bian Ji Bu 31: 893-897, 2011 (In Chinese). 
8. Bai J, Sun HF and Chen XF: Studies on the anti-mycobacterium tuberculosis activity of 4 Chinese medicinal herbs. Shi Zhen Guo Yi Guo Yao Bian Ji Bu 18: 77-78, 2007.

9. Liu MX, Sun FJ, Wang CX, et al: Effects of the extract of Coptidis Decoction for detoxification in MDR model rats on cell apoptosis and correlative study. Zhong Yao Cai Bian Ji Bu 32: 1270-1272, 2009 (In Chinese)

10. Li JG, Gao AL, Liu XH and Li NN: The effect of crewels pills on protection and pathological changes degree of internal organs for mouse suffering MDR-TB. He Nan Zhong Yi Xue Yuan Xue Bao Bian Ji Bu 23: 28-29, 2008 (In Chinese).

11. Guo XM, Hong YH and Zhou ZL: Advances in studies on chemical constituents from plants of Ranunculus. Zhong Cao Yao Bian Ji Bu 32: 748-750, 2001 (In Chinese).

12. Wang AW, Wang M, Yuan JR, et al: The study on antitumor effects in vitro of different extracts in Radix Ranunculi Ternati. Tian Ran Chan Wu Yan Jiu Yu Kai Fa Bian Ji Bu 16: 529-531, 2004 (In Chinese)

13. Liu XL, Zhang Y and Liu XL: Anti-coxsackievirus B3 effects of Sophora flavescens alkaloids in vitro. Shen Yang Yao Ke Da Xue Xue Bao Bian Ji Bu 23: 724-730, 2006 (In Chinese).

14. Liu D, Jiang YP and Cheng ZL: Sheltering effect on acute and chronic liver injury of mice due to drug combination of glycyrrhizin with matrine to carbon tetrachloride. 30: 581-582, 2007.
15. Feng L, Jia XB, Cheng Y and Li X: Advances in chemical constituents of Prunella vulgaris and antitumor mechanisms. Zhong Hua Zhong Yi Yao Xue Hui She 23: 428-434, 2008 (In Chinese).

16. Zhang MZ and Wang XQ: Effects of extract from Prunella vulgaris combined with chemotherapeutic agents on proliferation of lymphoma cells. Zhong Liu Bian Ji Bu 299: 961-964, 2009 (In Chinese).

17. Fang X, Chang RC, Yuen WH, et al: Immune modulatory effects of Prunella vulgaris L. Int J Mol Med 15: 491-495, 2005.

18. Yang K, Wang YS, Wang LP, et al: Brief review and clinical analysis of pharmacological action of Stellera chamaejasme L. Yi Xue Xin Xi Bian Ji Bu 23: 2496-2498, 2010 (In Chinese).

19. Wu SZ and Song JF: Mycobacterium tuberculosis pathogenesis and immunity. Zhong Hua Jie $\mathrm{He} \mathrm{He} \mathrm{Hu} \mathrm{Xi} \mathrm{Za}$ Zhi Bian Ji Bu 26: 101-103, 2003 (In Chinese).

20. Cole ST, Brosch R, Parkhill J, et al: Deciphering the biology of Mycobacterium tuberculosis from the complete genome sequence. Nature 393: 537-544, 1998.

21. Cooper AM, Dalton DK, Stewart TA, et al: Disseminated tuberculosis in interferon- $\gamma$ gene-disrupted mice. J Exp Med 178: 2243-2247, 1993

22. Zhan L, Dai HC, Yang ZP, et al: The study of expression of SHSP and GLS in peripheral blood lymphocytes of drug resistant tuberculosis patients with ternatolide. Zhong Guo Zhong Yao Za Zhi She 27: 677-679, 2002 (In Chinese). 